\title{
Utility of Small Molecule TLR4 Antagonist (+)-Naltrexone in Suppression Of Preterm Birth Induced In Mice By Platelet Activating Factor
}

\author{
Sarah Roberston ${ }^{1}$, Hanan Hamimi Wahid ${ }^{2}$ \\ ${ }^{1}$ Robinson Research Institute, School of Medicine, The University of Adelaide, \\ Australia \\ ${ }^{2}$ Department of Basic Medical Science, Kulliyyah of Medicine, International Islamic \\ University Malaysia
}

Presenter: Hanan Hamimi Wahid

Introduction: Microbial and sterile triggers of inflammation can activate Toll-like receptors (TLR) to initiate synthesis of pro-inflammatory cytokines that in turn drive the parturition cascade leading to preterm delivery (PTD). Platelet activating factor (PAF) has been identified as a key endogenous sterile inflammatory trigger that is elevated in the amniotic fluid in both normal and PTD in human. Intrauterine administration of PAF in mice results in PTD. We investigated the role of TLR4 in PAF-induced PTD. Materials and Methods: BALB/c females were administered PAF or vehicle i.p on gestational day (gd) 16.5 , followed by treatment with the small molecule TLR4 antagonist, (+)-Naltrexone, or vehicle control. Mice were observed for PTD, and in the absence of PTD mice were killed on gd 18.5 and implantation sites, fetal viability and fetal and placental weights were determined. Results: PAF administration lead to PTD in 64\% (9/14 mice), with reduced numbers of viable fetuses compared to vehicle controls. (+)Naltrexone administration to mice given PAF was able to prevent PAF-induced PTD and viable fetuses were comparable in number to control groups. Secondly, wildtype and Tlr4-/-BALB/C female mice were mated to males of the same genotype, and were challenged with PAF or vehicle on gd 16.5. Tlr4-/- females had $17 \%$ (2/12 mice) of PTD, with the number of viable fetuses similar to wildtype females when administered with PAF, suggesting that PAF may activate TLR4independent pathways to drive fetal inflammatory injury. Conclusion: PAF is a clear mediator of inflammation-driven PTD, through TLR4-dependent and TLR4independent pathways. 